\title{
Differentiated Approach in Reconstructive Surgery of Large Defects of The Anterior Wall of The Trachea
}

\author{
AA Kayumhodjaev, RY Hayaliev*, OD Eshonkhodjaev and ShN Khudaybergenov \\ SI RSSPMCS named after acad V Vakhidov, Uzbekistan \\ *Corresponding author: RY Hayaliev, SI “RSSPMCS named after acad V Vakhidov", Tashkent, Uzbekistan
}

\begin{tabular}{ll}
\hline ARTICLE INFO & ABSTRACT \\
$\begin{array}{l}\text { Received: } \\
\text { Published: February 07, } 2019\end{array}$ & $\begin{array}{l}\text { Citation: AA Kayumhodjaev, RY H, OD Eshonkhodjaev, ShN Khudaybergenov. Differenti- } \\
\text { ated Approach in Reconstructive Surgery of Large Defects of The Anterior Wall of The } \\
\text { Trachea. Biomed J Sci \& Tech Res 15(1)-2019. BJSTR. MS.ID.002630. }\end{array}$
\end{tabular}

\section{Introduction}

Plastic surgery of extensive defects of the cervical trachea involves the restoration of not only soft tissue defect, but also mucosa and tracheal skeleton function [1-7]. This fact requires an sufficient supply of full-fledged devoid of hairs skin in the reconstruction zone. The absence of such areas near the tracheal defect (sharp dystrophic or scar changes) requires the use of tissues with skin taken from other areas of the patient's body (the formation of the Filatov stem, the movement of skin-fascial flaps on the vascular pedicle, autotransplantation of complex flaps on microvascular anastomoses) [2-4]. A large number of possible tissues and materials, as well as options for operations aimed at eliminating the defect of the cervical trachea are proposed $[2,4,6,8]$. The main requirements for them are clearly formed: they must be of sufficient size, provide a frame function and the possibility of evacuation of the secret, as well as be resistant to infection $[5,6,9]$. Despite this, the problem is relevant and continues to be the subject of research and discussion of thoracic surgeons, plastic surgeons and otolaryngologists $[4,12,13]$.

The choice of a method for eliminating a tracheal defect depends on its size, the depth of the airway lumen and the state of the surrounding tissue defect $[1,10,11]$. With a small size of the defect, a depth of the respiratory tract more than $1.5 \mathrm{~cm}$, and an unexpressed scar process around the defect, it is possible to use local plastics $[1,3,7,11]$.

\section{Material and methods}

In the department of surgery of lungs and mediastinum of SI "RSSPMCS named after acad. V. Vakhidov" in the period from 2008 to 2018,103 patients with defects of the anterior wall of the cervical trachea were hospitalized. In case of small-sized defects, skinmuscle-skin plastic surgery was made by Bokstein method in the modification of our clinic. In the case of insufficiency of the seams in local plastic, restenosis and large size of the defect of the anterior wall of the trachea, we used plastic operations with moved flaps, including implantation of the autorib cartilage graft. When the three-layer skin-muscle plastic stitching of the muscles as a second layer often leads to a narrowing of the lumen of the trachea and reduce the depth of the airway in the region of the defect. The significant scar process of surrounding the defect soft tissues prevents the approach of the wound edges. In this case, the solution to the problem may be the use of displaced flaps from areas nearby the defect. The most acceptable are: side, top-side and supraclavicular flaps. In 10 patients with a large defect in the anterior-lateral walls of the trachea and the distal part of the larynx plastic operation with the use of displaced flaps on the vascular pedicle were performed. In 2 cases with defect sizes of more than $5 \times 3 \mathrm{~cm}$, plastic operation was performed with the use of a pre-fabricated cutaneous fascial cartilage delta-pectoral flap on the vascular pedicle (Table 1). The use of the displaced skin and fascial flaps on a vascular pedicle for 
closure of the defect of the trachea can allow to restore the mucous membrane region of the defect of the trachea without the use of Table 1: Plastic defect of the trachea is displaced flaps. surrounding scar tissue and close the defect with a second flap with no tension on the wound edges.

\begin{tabular}{|c|c|c|c|}
\hline \multirow{2}{*}{ Type of plastic } & \multicolumn{2}{|c|}{ Used flaps in plastic of: } & \multirow{2}{*}{ Number } \\
\cline { 2 - 4 } & mucosa lining of the trachea (first layer) & the wound defect (second layer) & side flap \\
\hline Plastic with two moved flaps & upper-side flap & free skin graft & 3 \\
\hline Displaced flap + free skin graft & the supraclavicular flap & the supraclavicular flap & 3 \\
\hline Closure of the defect + displaced flap & skin flap with the edges of the defect & free skin graft & 2 \\
\hline Deltopectoral flap (DPF) + auto costal cartilage & DPF skin+cartilage & 3 \\
\hline
\end{tabular}

\section{Clinical Example}

\section{Patient N. 26 Years Old Diagnosis}

Defect of the anterior wall of the trachea. Postintubation, posttracheostomy duplex cicatricial stenosis of the trachea (tracheolaryngeal region and the middle third). Condition after plasty of the trachea on the T-shaped stent (19.09.17). Condition after skin-muscle plasty of the lower part of the defect of the anterior wall of the trachea with leaving the upper part of the stoma on the T-shaped stent (04.04.18). The patient's tracheal defect was eliminated in 2 stages. Initially, a skin-fascial supraclavicular flap on the left and a circular flap around the defect were formed (10.10.2018). We raised the left supraclavicular and paratracheal skin-fat flap and back sewn with the abandonment of rubber graduates ("biological hardening of the flap") (Figure 1). The next stage - plastic of the anterior wall of the trachea by previously formed cutaneo-fascial flaps (16.10.18). The edges of the previously formed circular flap were isolated to form the neo wall of the trachea. The sutures of the previously formed supraclavicular skin-fascial flap on the left were excised, flap with good blood supply, moved to the defect area without tension to close the tracheal defect with the second layer (Figure 2). The control TBFS in the dynamics - the leather insertion is determined on the front wall of the trachea $2 \mathrm{~cm}$ below the vocal cords, hermetically. The lumen of the trachea in the affected area is wide (Figure 3). Plastic of extensive defects of trachea by prefabricated delto-pectoral flap on a vascular pedicle.

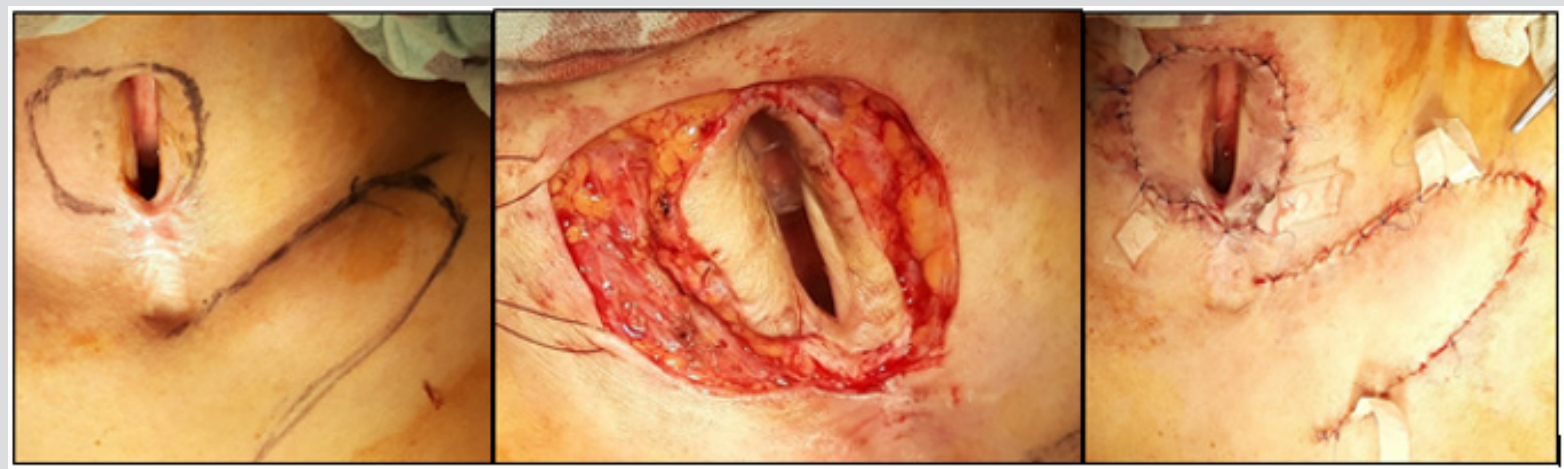

Figure 1: Stage 1- formation of flaps.

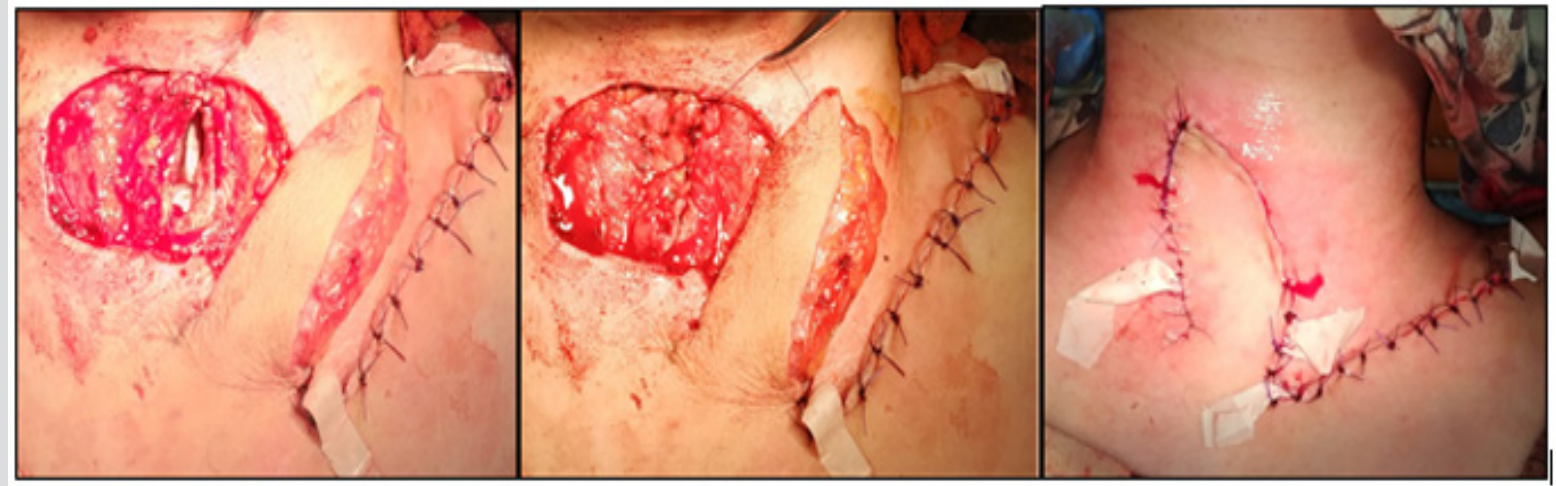

Figure 2: 2-nd stage - plasty of the defect of the trachea. 


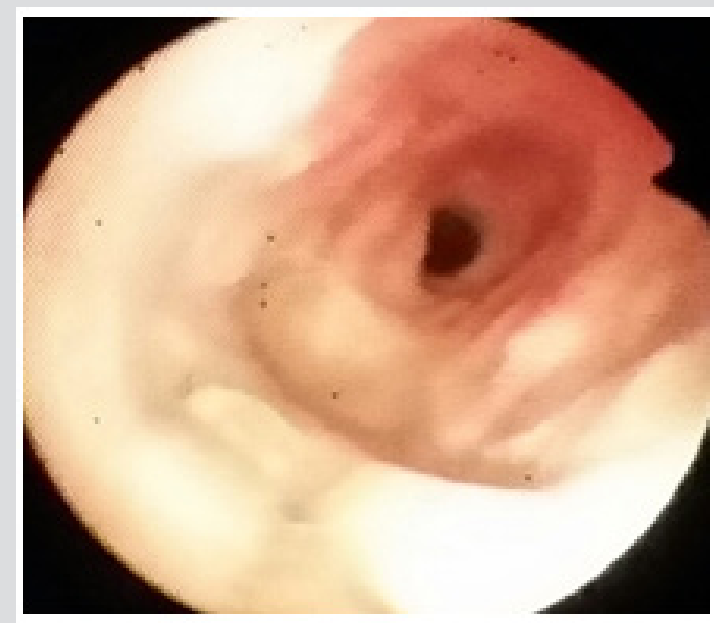

Figure 3: The same patient. Control TBFS on the 10-th day after surgery.

\section{Patient P. 28 years, with a Diagnosis:}

Long posttracheostomy, postoperative cicatricial stenosis of the upper and middle third of the trachea. Extensive scar defect and deformation of the anterior wall of the trachea and soft tissues of the neck. Scar stenosis of the left main bronchus. Bronchiectasis of both lungs. Anamnesis: in 1992, at the age of 2 years, she was hospitalized in the regional hospital in Fergana with inflammatory process of lungs. Intensive care for 7 days, tracheostomy. In subsequent patient since 1992 up to the present time has undergone multiple surgeries because of the scar stenosis of the trachea in Moscow, in Khabarovsk, in the otolaryngology department of the Tashkent State Medical Institute. MSCT of the chest from 09.06.2016 - an irregular thickening of the walls of the trachea to 8-10 $\mathrm{mm}$ with narrowing of the lumen throughout a minimum diameter of $9 \mathrm{~mm}$. Stenosis of the left main bronchus to $5.8 \mathrm{~mm}$. Semi-rings of the trachea calcified. Bronchiectasis of both lungs with peribronchial infiltration. Due to the fact that the patient had multilevel tracheal stenosis, the defect was large and in the middle of the defect there was a connective tissue septum on the posterior wall, first it was necessary to install a T-shaped stent to expand the middle part and align the tracheal axis (Figure 4). It took about 1.5 months. Due to the presence of extensive defect of the anterior wall of the trachea and soft tissues of the anterior surface of the neck, plastic was performed by a prefabricated Delta-pectoral flap. The size of the defect was $3 \mathrm{sm}$ wide and $8 \mathrm{sm}$ high, the depth of the lumen was $1.2 \mathrm{sm}$. The axis of the airway was broken, the distal division of the larynx and upper third of the trachea in the projection of the defect had the direction of the axis from behind forward in the sagittal direction, and the middle third of the trachea in a retrosternal region had the direction of the axis from front to back in the sagittal direction. We have improved the method of forming a pre-fabricated flap. As a reference tissue in this case we had used the implanted in the flap autorib cartilaginous skeleton. Depending on the parameters of the tracheal defect, a part of the cartilage was taken in the area of connection of VI and VII ribs, according to the generally accepted technique in plastic surgery. Subsequently formed cartilage framework implanted subfascial in the distal portion of the delto-pektoral flap.

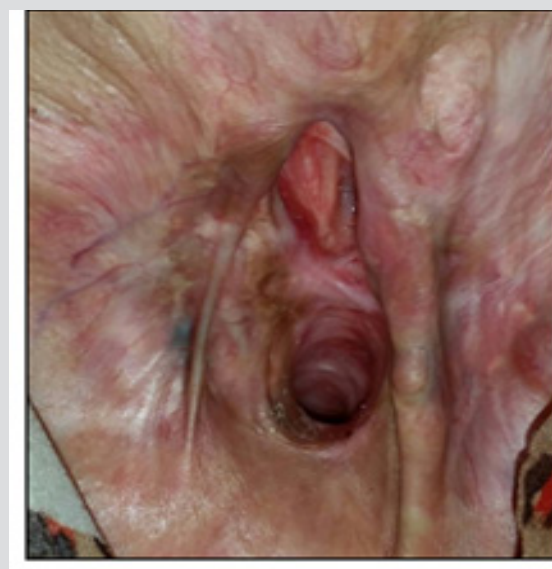

a

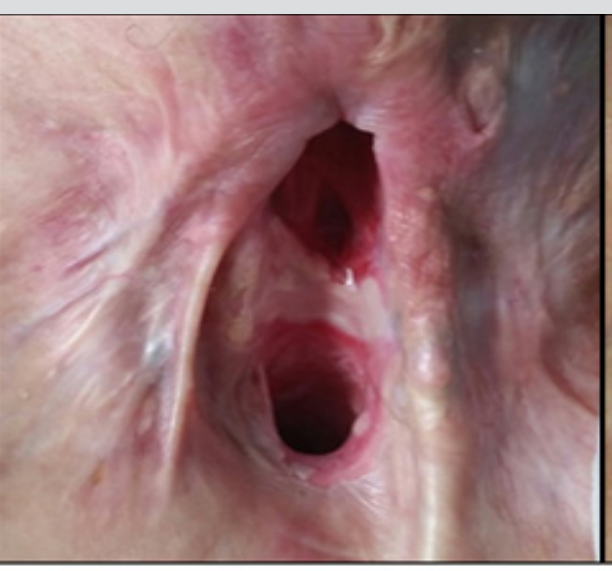

b

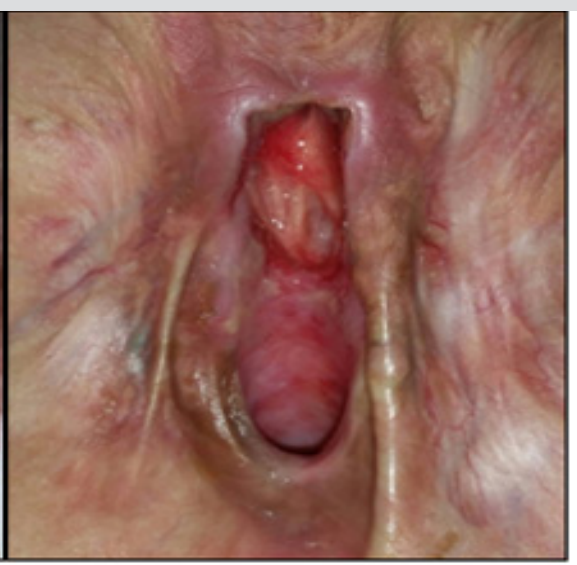

c

Figure 4: Multifocal scar stenosis of the trachea. Extensive scar defect of the anterior-lateral walls of the trachea and soft tissues of the neck (a). A week after the installation of the T-stent (b). After 6 weeks (c).

\section{Stage of Operation}

\section{Stage 1}

The formation prefabricating deltopectoral flap with implantation Autorib cartilaginous skeleton in the distal part of the flap
(Figure 5). No data for restenosis were observed for a long period of follow-up. It was decided to perform the 2nd stage of the operation-the movement of the previously prepared skin-cartilage flap with the closure of the lower $2 / 3$ of the tracheal defect with the formation of the mucous insert. 


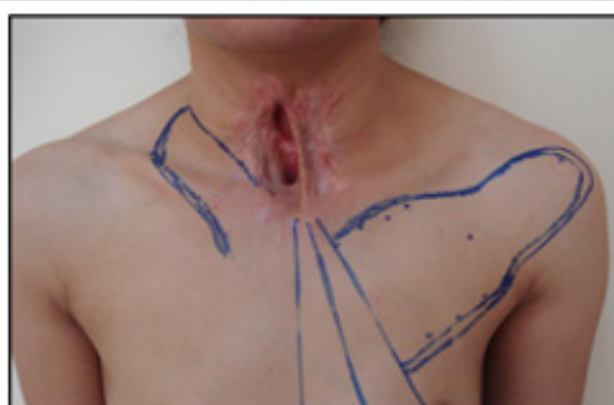

a

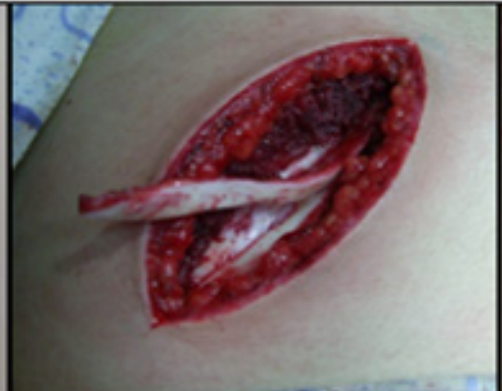

b

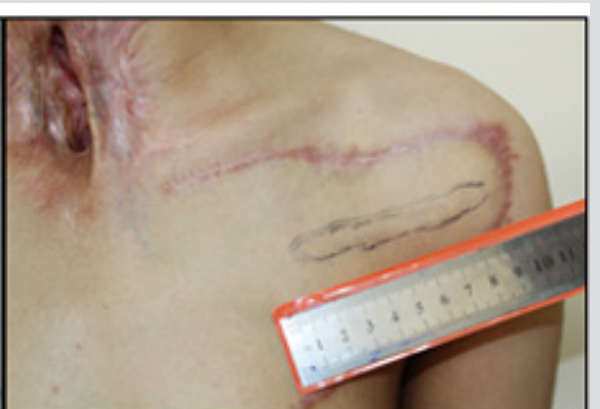

C

Figure 5: Deltopectoral flap marking (a). Preparation of rib cartilage (b). Condition after the implantation of costal cartilage graft (c).

\section{Stage 2}

Raising the pre-fabricated flap with the formation of a round stem. The substitution of the defect of the trachea with the restoration of the mucosal lining (leather flap) and cartilage of skeleton (due to implanted autorib cartilage). Closure of the donor area and the fascial part of the flap with split skin (Figures $6 \& 7$ )

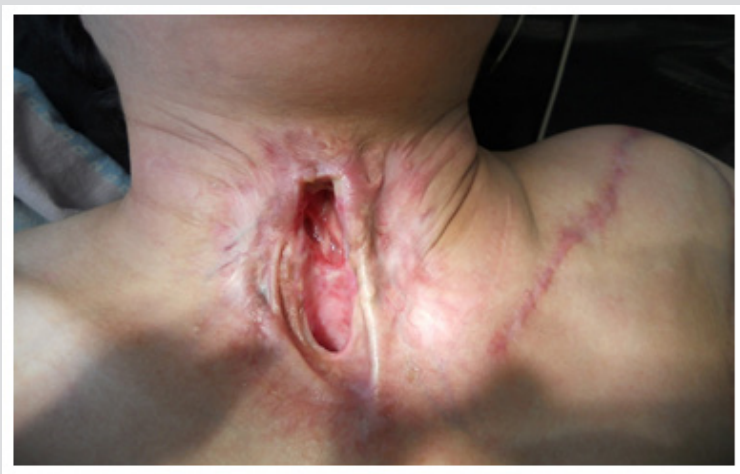

Figure 6: A month after the pre-fabrication of a deltopectoral flap with implanted cartilage before lifting and rotating the flap.

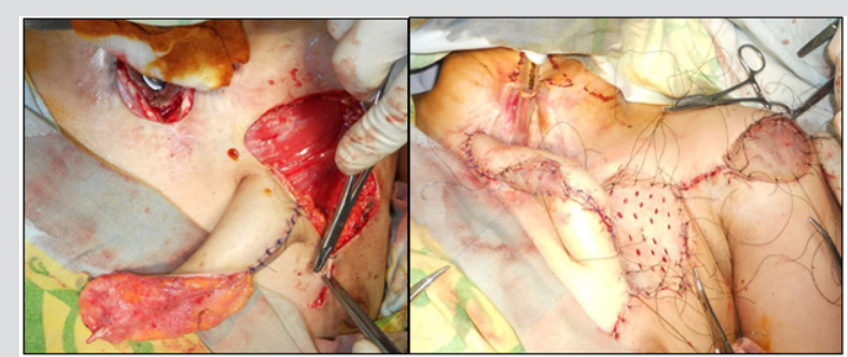

Figure 7: Raising a previously formed pre-fabricated flap. Suturing of the tracheal defect.

\section{Stage 3}

The final plastic of the tracheal defect (Figures 8 \& 9). Biological training of the flap followed by cutting off it's leg with suturing along the axis of the trachea from the left side and strengthening the frame by autocartilage. 22.12.2018 partially plastic defect of the trachea by pre-prepared skin-fascial cartilage flap was performed. The postoperative period was without complications. Primary healing of wound. Sutures were removed on the 14th day after surgery. Diagnostic TBFS - trachea is wide, stable. The patient was observed for a long period, data for restenosis were not observed. 11.01.2009 plastic of the residual defect of the cervical trachea under local anesthesia was made by suturing the fabricated Delta-pectoral flap on the right side with full closure (Figure 9). When performing MSCT, a wide lumen of the trachea is visualized with a deformation in the middle third, which does not significantly affect the patency. Inside the moved flap, an autorib cartilage is traced, running diagonally from left to right, crossing the longitudinal axis of the formed anterior wall of the trachea (Figure 10).

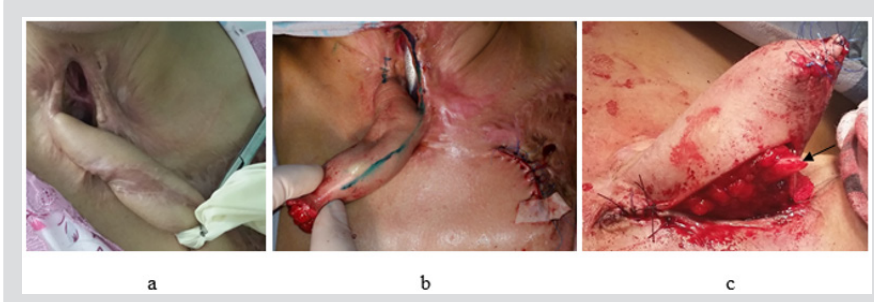

Figure 8: Biological training of the flap (a). Amputation of the flap (b). Suturing the flap along the axis of the trachea from one edge (c). The arrow shows the implanted autoccartilage.

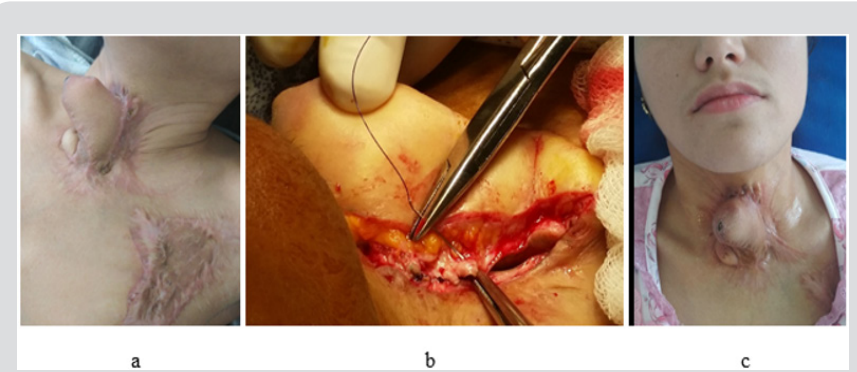

Figure 9: Complete closure of the tracheal and soft tissue defect in 2 weeks. Before surgery (a). Suturing the flap on the right side (b). Extensive defect is completely closed by the flap. 


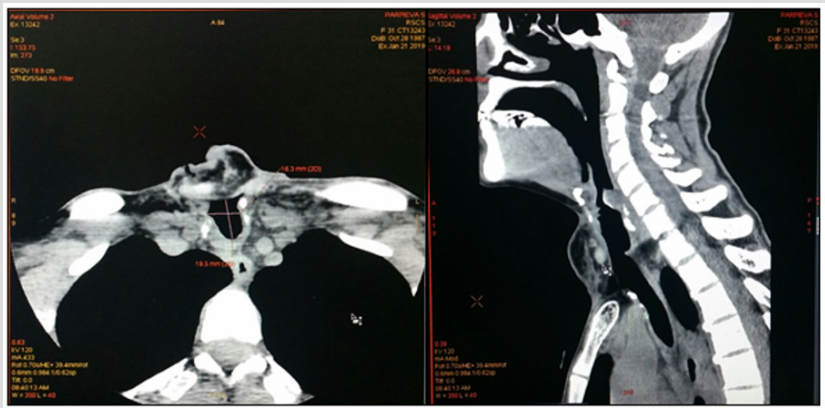

Figure 10: MSCT of the trachea after 3-stage operation.

On bronchoscopic control the tracheal lumen is also wide with a deformation in the middle third, up to $11 \mathrm{~mm}$ in diameter in the narrowest place. In the future, it is planned to cut off excess flap tissue.

Thus, thanks to the multi-stage plastic surgery, we managed to eliminate the extensive defect of the trachea and soft tissues, because of which patient suffered for 26 years and was limited both physically and mentally, and the result is undoubtedly the main basis for improving the quality of life. Thus, the choice of the method of plastic surgery or reconstruction of the tracheal defect is determined in each case individually. When the size of the defect of the trachea up to $2 \times 4 \mathrm{~cm}$, a sufficient depth of the lumen of the trachea corresponding to the clearance above and below the defect, a single axis of the trachea and the distal part of the larynx without displacement and deformation of the lumen the skin-muscle plasty of a defect with the substitution of local tissues is used. Rotary skinfascial flaps can be used, including those with pre-implantation of the cartilage frame. The indication for the use of displaced flaps for plasty of the tracheal defect is a satisfactory depth (more than 1.5 $\mathrm{sm}$ ) and the size of the defect is not more than $4 \times 2 \mathrm{sm}$.

With a significant scar process around the defect (as a result of repeated surgical interventions) and the inability to use the method by Bokstein, using skin-fascial displaced flaps on the vascular pedicle is effective.

The use of displaced flaps allows: to form a mucous membrane in the defect area without tension of the lateral walls of the trachea (the first layer); to close the defect (or to cover the cartilaginous implant) with a well blood-supplied second flap. A complex-compound prefabricated flap (Delta-pectoral skin-fascial-cartilaginous flap) can be used in the presence of a persistent defect and formed tracheal lumen at this level, without signs of restenosis of the respiratory tract, in the absence of inflammation and infection of tissues around the defect. When using a moved flap to form a mucous lining in the area of the tracheal defect, it is advisable to use the first stage of biological training of this flap, which consists in its separation and re-fixation by seams to the mother bed. The task of this stage is to strengthen the axial circulation and increase the survival of the flap. The main stage is performed on 12-14 days after the first stage.

\section{References}

1. Amirov FF (1956) Plastics of defects of the trachea and bronchi. Exper hir 2: 47-52.

2. Gudowsky LM, Milanov NO, Parshin VD, Trofimov EI (2006) Long-term results of elimination of extensive defects of the trachea with the aid of microsurgical technology Problems. Tuberculosis and dis. of lungs p. 1823.

3. Zenger VG, Nasedkin AN, Parshin VD (2007) Surgery of lesions of the larynx and trachea. M.: Medicine p. 364.

4. Milanov NO, Trofimov EI, Parshin VD (2006) Closure of large defects of the trachea using revascularizing prefabricating radia skin and cartilage autograft. Annals of plastic. Reconstr. and aesthetic. Surgeries № pp. 109.

5. Milanov NO, Trofimov EI, Parshin VD, Shimbireva O Yu (2007) Free revascularizable grafts in surgery of the trachea. Issues of reconstructive and plastic surgery № 3: 79-82.

6. Parshin VD, Milanov NO, Gudowsky LM, Trofimov EI, Zelenin AS, et al. (2008) Ten years of application of microsurgical techniques in reconstructive surgery of the trachea. Thoracic and cardiovascular surgery 2: 36-42.

7. Parshin VD, Porkhanov VA (2010) Surgery of the trachea with operative surgery. Aldi-Print pp. 387.

8. Topolnitskiy EB, Dambaev GC, Semichev EV, Shefer NA, Gunther VE (2010) Method of substitution graduate of defects of the trachea and larynx. Patent for invention.

9. Allison K Royer, Mark C Royer, Jonathan Y Ting, Edward C Weisberger (2015) The use of a prefabricated radial forearm free flap for closure of a large tracheocutaneous fistula: a case report and review of the literature. Journal of Medical Case Reports. December 9: 251-253.

10. Gebauer PW (1951) Reconstructive surgery of the trachea and bronchi: late results with dermal grafts. J thorac Surg 22(6): 568-584.

11. Grimmer JF, Gunnlaugsson CB, Alsberg E (2004) Tracheal reconstruction using tissue-engineered cartilage. Arch. Otolaryngol. Head Neck Surg 130: 1191-1196.

12. Jianxing $\mathrm{He}$, Xin $\mathrm{Xu}$, Manyin Chen, Shuben $\mathrm{Li}$, Weiqiang Yin et al. (2009) Novel Method to Repair Tracheal Defect by Pectoralis Major Myocutaneous flap. Ann Thorac Surg 88: 288-291.

13. Yui Watanabe, Tadashi Umehara, Aya Harada, Soichi Suzuki (2015) Successful closure of a tracheocutaneous fistula after tracheostomy using two skin flaps: a case report. Surgical Case Reports 1: 43-47. 
ISSN: 2574-1241

DOI: 10.26717/BJSTR.2019.15.002630

RY Hayaliev. Biomed J Sci \& Tech Res

(c) (i) This work is licensed under Creative

Submission Link: https://biomedres.us/submit-manuscript.php

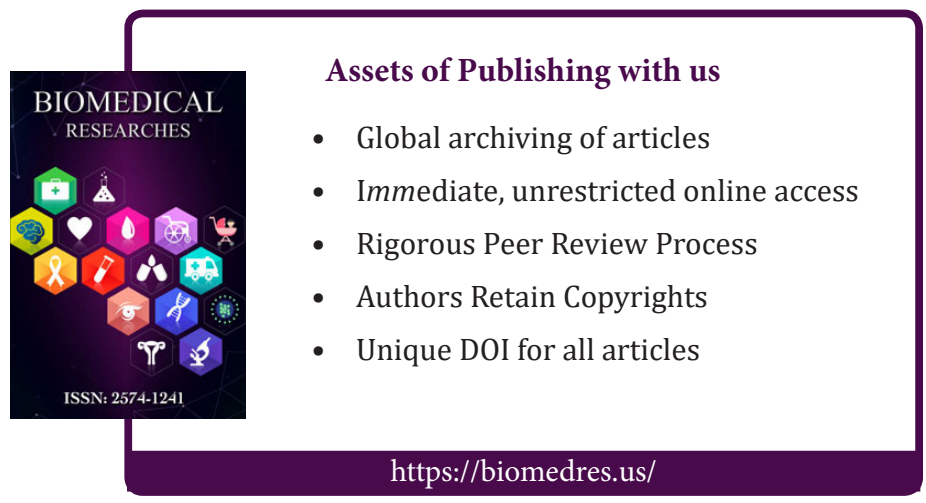

